\title{
Study on the Design of A High-precision Indoor Positioning System
}

\author{
Lei $\mathrm{Li}^{1}$ \\ ${ }^{1}$ Xi'an University of Electronic Science and Technology, Xi'an, 710071 \\ 346591653@163.com
}

KEYWORDS: Indoor Positioning; DW1000; UWB Precision

\begin{abstract}
Firstly, explained the situation and development trend of indoor positioning technology, and discusses the use of UWB signal indoor positioning technology to achieve the principle of further argues that the analysis of the indoor positioning system solutions. By combining the latest technology of the moment, creatively put forward to the DW1000 DecaWave chip-based, technology-based indoor positioning of UWB DW1000 and TI's MSP430F5529 combination. Finally, the preparation of the client "TAG" and server "ANCHOR" software program using MATLAB GUI simulated indoor positioning environment, to achieve high accuracy indoor positioning system based on the DW1000.
\end{abstract}

\section{Introduction}

With the improvement of people's living standards, the location information in people's daily life and plays an important role. And as one of LBS (location based services) core technology, indoor positioning is an important part, it can be widely used for indoor navigation, security, fire and so on. In recent years, technology and related services industry forward position to provide ubiquitous indoor develop location-based services, the main driver of indoor location-based services can bring great applications and commercial potential. Many companies, including OS providers, service providers, equipment and chip providers are in competition in this market.

With performance more new mobile devices such as mobile phones, tablets, and other wearable devices, networking equipment based on the rapid growth and proliferation of location-aware applications, location-aware play an increasingly important role. In the indoor and outdoor environment, continuously provide reliable location information can provide users with a better user experience. Outdoor positioning and location-based services has matured, based on GPS and map location services are widely used, and a variety of mobile devices has become one of the most widely used. With the outdoor positioning technology matures, stability and precision indoor positioning more and more people's attention, so indoor positioning technology has broad prospects for development.

2010, Microsoft will be the map to start adding some indoor shopping malls and airports map; 2011 Google also its Android phones Google Maps adds 10,000 indoor places map nine countries. According to the survey agency IMS7月的报告称, there are at least 130 companies involved in this field, including the indoor maps, indoor positioning, indoor track, indoor navigation and other research and development. The report predicts that by 2016 there will be nearly 120,000 indoor places maps for users. In addition, the founder and chief CEONathanPettyjohn Aisle411 think, 2-3 years, indoor maps market will reach \$ 4.7 billion. 2013, Google, Apple, Baidu and many other giants have begun eyeing the indoor location: Google indoor maps quickly covering North America, 
Europe, Australia and Japan, more than 10,000 large venues, and the total number is still growing: Baidu also launched its own indoor positioning, now covers Beijing number of shopping malls.

\section{The UWB Positioning Technology based on DW1000}

\section{A. Two-way ranging algorithm}

In DW1000 module, each transmit or process information received, the corresponding register will automatically record when sending or receiving a time stamp, the time stamp "TimeStamp" after a certain operation (or send an adjacent transceiver time mark received subtraction) can be obtained from a radio signal to another piece DW1000 DW1000 transmission time TOF, we will be multiplied by the speed of light can be obtained by TOF distance between two DW1000.

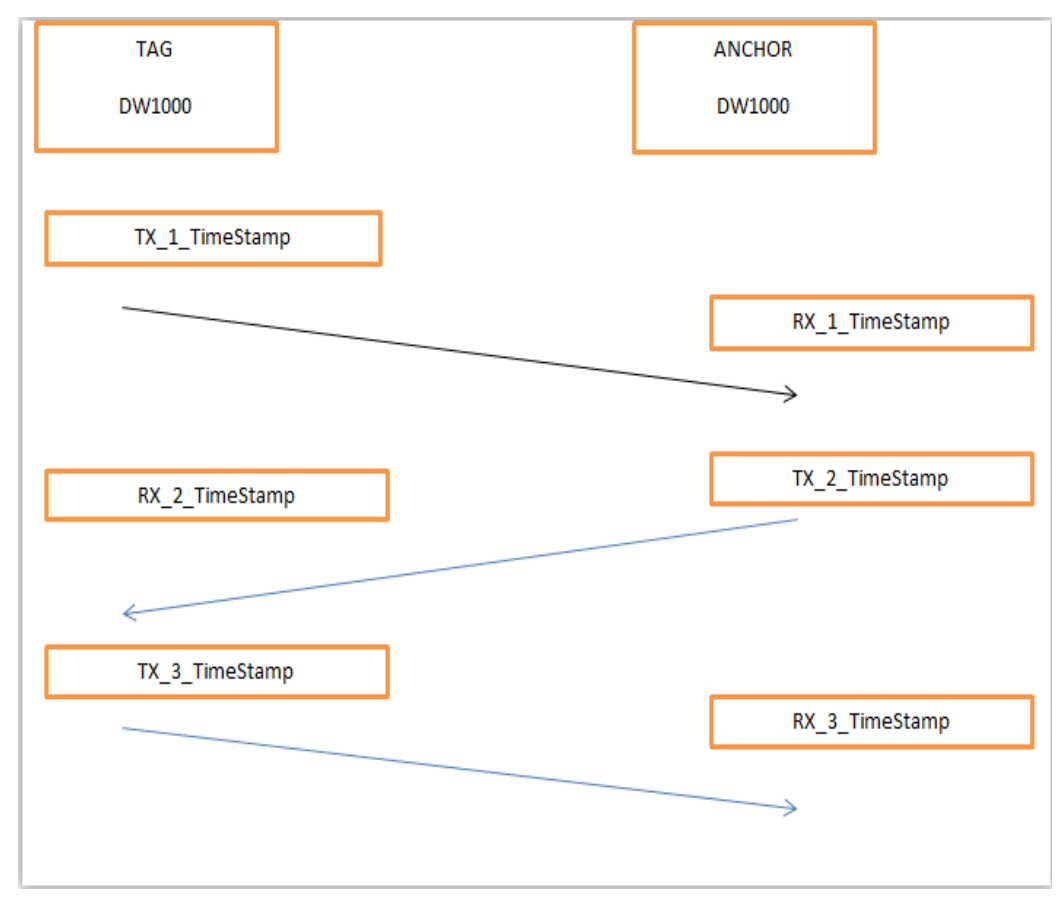

From the above chart shows:

Figure 1 two-way ranging algorithm

TOF $=\left(\left(R X \_2-T X \_1\right)-\left(T X \_2-R X \_1\right)+\left(R X \_3-T X \_2\right)-\left(T X \_3-R X \_2\right)\right) / 4$;

DISTANCE $=$ TOF $*$ (the speed of light);

\section{B. The Design Method of the System}

With the above DW1000 way ranging algorithm as the theoretical basis, we will further distance between points rise for the design of dynamic positioning between multiple points. Due to the spatial distribution of a variety of indoor, here we rectangular distribution of conventional indoor design case, exploring positioning algorithm design rectangular interior space. We will be placed in three DW1000 chip three corners of the rectangular chamber, which three DW1000 as server-side "ANCHOR", for signal detection "TAG" and sent from the distance. There is a need for additional DW1000 as a client "TAG", "TAG" in the actual positioning often changing positions, and "ANCHOR" interactive information with other three. Achieve real-time positioning information exchange "TAG" and "ANCHOR" constantly in. 


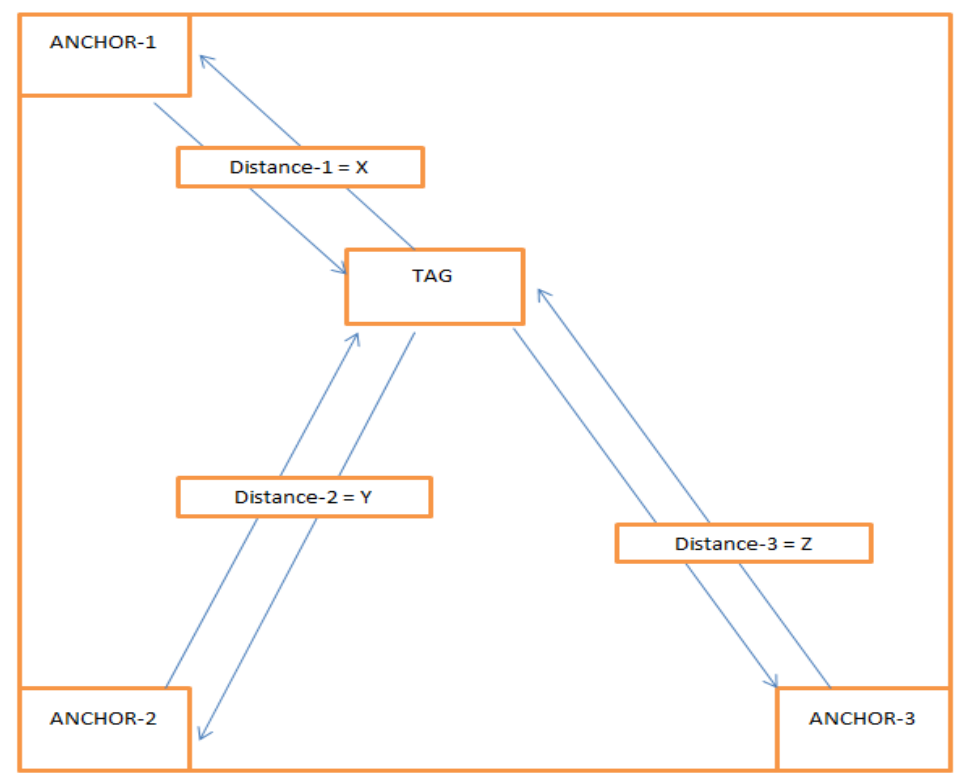

Figure 2 rectangular indoor location model

As shown above, when we know the length of the rectangular chamber $A$, the width $B$, we can use the geometric method measured "TAG" real-time coordinate $(a, b)$.

$$
\begin{aligned}
& \mathrm{a}=(\mathrm{Y} * \mathrm{Y}-\mathrm{Z} * \mathrm{Z}+\mathrm{A} * \mathrm{~A}) /(2 * \mathrm{~A}) \\
& \mathrm{b}=(\mathrm{Y} * \mathrm{Y}-\mathrm{X} * \mathrm{X}+\mathrm{B} * \mathrm{~B}) /(2 * \mathrm{~B})
\end{aligned}
$$

When confronted with the interior space distribution of irregular shape, we can calculate the coordinates using the least squares method. The basic idea of the least square method is: According to various "ANCHOR" Device A, B, C, ... received positioning device "TAG" time stamp value can be obtained from the "TAG" and various "ANCHOR" between, according to the each "ANCHOR" coordinates a (a1, b1), B (a2, b2), C (a3, b3), ... corresponding to the obtained matrix calculation "TAG" current location.

C. The Desig Application of $n$ Indoor Positioning PC side

Matlab Matlab GUI is a graphical user interface that comes with edit controls, support for visual programming and program code editor, able to call general Matlab function, but also do some drawing and data processing operations.

In this article we use Matlab GUI design "DW1000 Real Time Syetem" PC end user applications. Both provide a visual operation, but also timely analysis and processing "TAG" end position information sent and displayed in real time. 


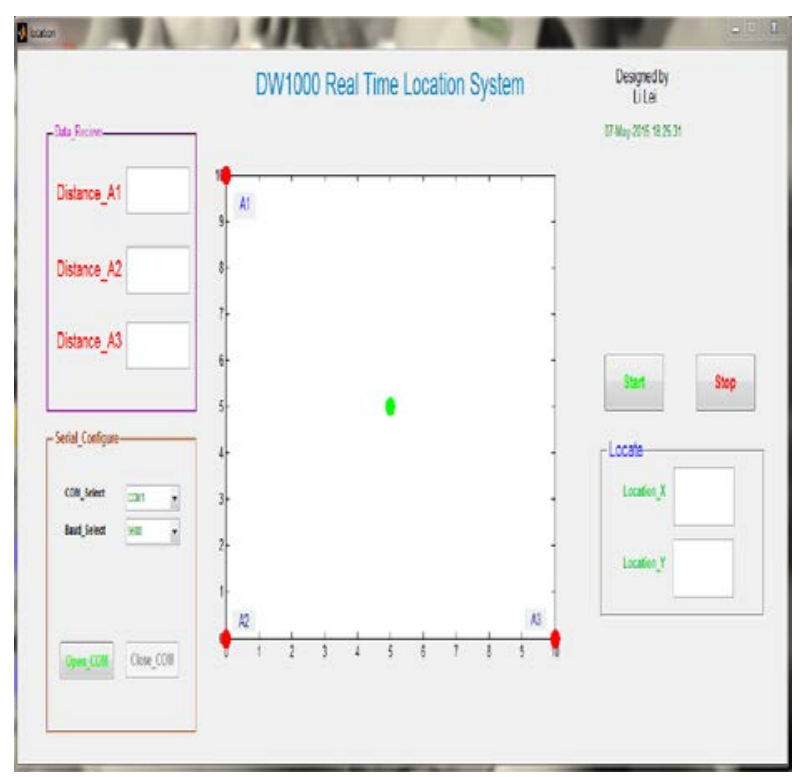

Figure 3 Indoor positioning application program interface

\section{System Functional Test and Data Analysis}

For demonstration of the real-time location system, it selected a medium-sized conference room as a test environment. The room is rectangular spatial distribution, relatively closed room, a long rectangular space is 6 meters wide and 10 meters. There are a number of tables and chairs inside, and randomly distributed.

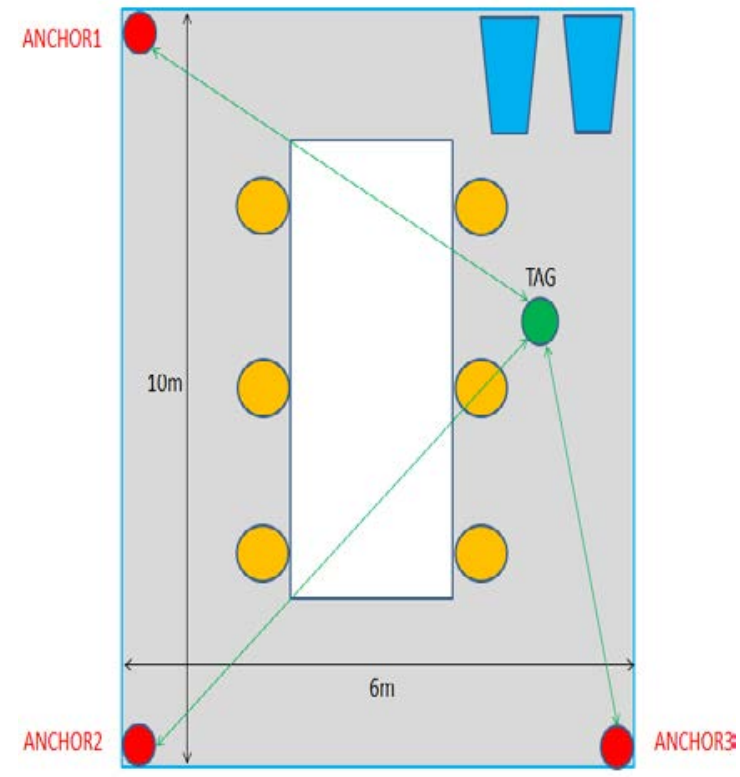

Figure 3 System Test Environment diagram

In order to achieve the two-dimensional positioning function, as shown above, the interior space with a schematic plan view of the method shown. We three DW1000 "ANCHOR" plate is fixed at three corners of the room, in the process of locating the "ANCHOR" plate can not move, their coordinates are as follows: $(0,10),(0,0),(6,0)$. And during the process can move in positioning "TAG" board, and observe the changes in the positioning software end position coordinates on the PC side.

After the start of the test, constantly moving position "TAG" plate, measuring its actual coordinates and displayed on the PC side recorded coordinate values, respectively, and then 
displays the true value of the measured values are compared, analyzed its positioning accuracy. Without regard for the impact of UWB signal transmitting and receiving people or objects.

Table 1 "ANCHOR" coordinate distribution table

\begin{tabular}{|c|c|c|c|}
\hline “ANCHOR” & 1 & 2 & 3 \\
\hline $\mathrm{X}$ & 0 & 0 & 6 \\
\hline $\mathrm{Y}$ & 10 & 0 & 0 \\
\hline
\end{tabular}

Comparative results show the true value of the coordinate values of 10 sampling points in the table below:

Table 2 DW1000 positioning coordinates and the actual coordinates of the comparison table

\begin{tabular}{|c|c|c|c|c|c|}
\hline Position & $X$ true Value & Y Ture Value & $x$ & $Y$ & $\operatorname{Gap}(m)$ \\
\hline 1 & 1.2 & 2.3 & 1.03 & 2.4 & 0.20 \\
\hline 2 & 1.6 & 2.3 & 1.57 & 2.45 & 0.15 \\
\hline 3 & 2.0 & 2.3 & 1.9 & 2.36 & 0.12 \\
\hline 4 & 2.0 & 3.0 & 2.2 & 3.1 & 0.22 \\
\hline 5 & 2.5 & 3.4 & 2.41 & 3.68 & 0.29 \\
\hline 6 & 3.4 & 3.6 & 3.33 & 3.56 & 0.08 \\
\hline 7 & 3.4 & 4.0 & 3.57 & 4.0 & 0.17 \\
\hline 8 & 3.8 & 4.5 & 3.8 & 4.63 & 0.13 \\
\hline 9 & 4.2 & 5.5 & 4.34 & 5.57 & 0.16 \\
\hline 10 & 4.5 & 6.0 & 4.57 & 6.13 & 0.15 \\
\hline
\end{tabular}

The above table shows that: DW1000 measurement error based indoor positioning system substantially less than $0.2 \mathrm{~m}$, indicating that the system has a high positioning accuracy. If Taking into account the impact of UWB signal transmitting and receiving people and objects, increase the number of "ANCHOR" board, the system can reduce errors, achieve more accurate indoor positioning system. 


\section{Summary of the Indoor Positioning System and Its Prospects}

In today's society, with a variety of mobile devices with the positioning terminal popularity of indoor positioning in people's daily lives play an increasingly important role. Compared to an outdoor location, indoor location has more room for development. Therefore, design and development of high-precision, high reliability and indoor positioning systems in the market, rapid promotion is particularly important. Prospects indoor positioning system discussed herein outlook as follows:

(1) To achieve three-dimensional positioning

Indoor positioning system as discussed herein to achieve a two-dimensional positioning, the face of increasingly complex reality, to achieve three-dimensional positioning is also very necessary. To achieve three-dimensional positioning, the system needs discussed in this article to add a "ANCHOR", three non-coplanar "ANCHOR" can measure an "TAG" of the three-dimensional coordinates. In the three "ANCHOR" center of the sphere, each "ANCHOR" and "TAG" distances of the intersection of three spherical radius of each sphere is the "TAG" space (three-dimensional) coordinates.

(2) Design of mobile device side APP Android platform and the iPhone platform

Since the DW1000 highly integrated, easy to carry, so the future can be installed into a variety of mobile terminal equipment, design and development of the corresponding APP application, with each DW1000 allows the body to be brought about by technological convenience. Such as mobile phones and watches DW1000 module simultaneously with each other and ranging, when the phone is some distance away from their own watches will alarm, in order to prevent the phone is lost or stolen.

(3) Indoor positioning towards a more popular indoor navigation

When you walk into a big mall, and sometimes it does not make you look at the signs you want to be within easy reach of a restaurant or a clothing store. Therefore, the use of high-precision positioning DW1000 achieve indoor navigation feature that allows you can easily reach every corner of the room. Every major mall or indoor unit can develop their own distribution model and indoor navigation system, when you walk into an indoor mall, the appropriate authority will put the navigation system software download prompts sent to you, so you can always download and use.

\section{REFERENCE:}

[1] DecaWave. DW10000 User Manual [R]. Ireland: DecaWave Ltd, 2013.

[2] DecaWave. DW10000 Datasheet [R]. Ireland: DecaWave Ltd, 2014.

[3] DecaWave. PRODUCT INFORMATION: DWM10000 [R]. Ireland: DecaWave Ltd, 2013.

[4] DecaWave. APPLICATION NOTE: APU001 [R]. Ireland: DecaWave Ltd, 2013.

[5] Li Jian, Liu Heping. A New Weighted Centroid Localization Algorithm in Coal Mine Wireless Sensor Networks[C]//Proc. Of the 3rd International Conference on Computer Research and Development, [S. 1.]: IEEE Press, 2011. 